\title{
Einladung zur ordentlichen Mitgliederversammlung
}

\section{der Deutschen Gesellschaft für Manuelle Medizin (DGMM)}

Sehr geehrte Kolleginnen und Kollegen,

liebe Mitglieder unserer Gesellschaft

ich lade Sie herzlich zu unserer Mitgliederversammlung am

Freitag, den 20.11.2020

Beginn: 17.15 Uhr

in 10117 Berlin, CCO-Auditorium Charite Campus Berlin, Chariteplatz 1

ein.

\section{Tagesordnung:}

1. Begrüßung durch den Präsidenten und Genehmigung der Tagesordnung

2. Feststellung der Beschlussfähigkeit und korrekten Einladung

3. Bericht des Präsidenten

4. Bericht des Schatzmeisters

5. Bericht der Kassenprüfer

6. Entlastung des Vorstandes

7. Wahl des Präsidenten

8. Wahl des Schriftführers

9. Wahl des Schatzmeisters

10. Wahl der Kassenprüfer

11. Verschiedenes.

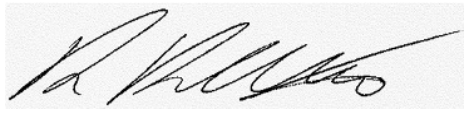

\section{Prof. Dr. R. Klett}

Präsident 


\title{
Forschungsberatungsstelle - Deutsche Stiftung Manuelle Medizin
}

\author{
Ausgabe Nr.03-2020
}

Sehr geehrte Leserin, sehr geehrter Leser,

mit diesem Newsletter möchten wir Sie über die aktuellen Forschungsthemen sowie über wissenschaftliche Neuigkeiten aus dem Bereich der Manuellen Medizin informieren. Zu jedem kurzen Beitrag finden Sie die entsprechende Literaturquelle zum Weiterlesen.

\section{Interexaminer reliability of thoracic motion palpation using confidence ratings and continuous analysis}

\section{Die Reliabilität der chiropraktische Diagnosetechnik ,Motion Palpation"}

Motion Palpation, d.h. die Palpation in bzw. von Bewegung, ist eine essenzielle chiropraktische Technik. Jedoch zeigen Reliabilitätsstudien nur ungenügende Übereinstimmungen zwischen den Untersuchern. Daher sollten die Praktiker dieser Studie zusätzlich ihre Zuversicht gegenüber dem von ihnen benannten Befund kundtun. Beurteilt werden sollte das anteriore sowie posteriore Gleiten von T3 bis T10 bei 52 asymptomatischen, studentischen Freiwilligen in Bauchlage. Die Untersucher gaben den Bereich der höchsten Einschränkung an, sowie ihre persönliche Einschätzung zur Sicherheit des erkannten Ergebnisses. Der Intrakorrelationskoeffizient bezüglich der genauen Lokalisationsübereinstimmung für alle Probanden war nur schwach (ICC $=0,311$ ). Jedoch war dieser gut (ICC $=0,827$, wenn nur die Ergebnisse bewertet wurden, bei welchem sich beide Rater sicher waren. Somit hat das „Confidence Level“/Zuversichtslevel einen deutlichen Effekt auf die Reliabilitätswerte der Untersucherübereinstimmung gegenüber der ausschließlichen Lokalisationsbeurteilung.

Quelle

Cooperstein R, Haneline M, Young M (2010). Interexaminer reliability of thoracic motion palpation using confidence ratings and continuous analysis. Journal of chiropractic medicine, 9(3):99-106. https://doi.org/10.1016/j.jcm.2010.06.004.

\section{The Spanish Osteopathic Practitioners Estimates and RAtes (OPERA) study: A cross-sectional survey}

\section{Querschnittsstudie zur Osteopathie in Spanien}

Das „Osteopathic Practitioners Estimates and Rates“-Projekt wurde 2018 durchgeführt, um eine Übersicht über das professionelle Profil der Osteopathen in Spanien zu erhalten. Insgesamt beteiligten sich 517 Osteopathen:

- $98 \%$ mit akademischen Abschluss - 75\% davon in Physiotherapie

- $61 \%$ gaben eine zusätzliche Tätigkeit neben der Osteopathie an

- $60 \%$ arbeiten 5 Tage/Woche, die meisten sehen dabei 21-30 Patienten/Tag (31\%), vorwiegend zwischen $18-40$ Jahren (67\%)

- $64 \%$ führen zu jeder Behandlung ein osteopathisches Assessment durch. $35 \%$ zumindest regelmäßig

- häufigste Untersuchungstechniken: Palpation in Bewegung, Palpation von Strukturen, Assessment von Tender-/Triggerpunkten, Assessment der viszeralen Mobilität, Assessment des Schädels

- $85 \%$ befürworten eine Regulierung der osteopathischen Profession

- $74 \%$ plädieren für die Osteopathie als Teil der Primärversorgung

Quelle

Alvarez G, Roura S, Cerritelli F, Esteves JE, Verbeeck J, van Dun PLS (2020) The Spanish Osteopathic Practitioners Estimates and RAtes (OPERA) study: A cross-sectional survey. PLoS ONE 15(6): e0234713. https://doi.org/10.1371/journal.pone.0234713.

\section{Potential mechanisms for lumbar spinal stiffness change following spinal manipulative therapy: a scoping review}

\section{Literaturrecherche nach möglichen Mechanismen des positiven Einflusses von Manipulation auf lumbale Beschwerden}

Obwohl bekannt ist, dass eine Manipulation lumbale Probleme reduzieren kann, ist nicht eindeutig geklärt, welcher Mechanismus dahinter liegt. Daher wurde eine Literatursuche in Medline bzw. Pubmed und CINAHL zwischen 2000 und Juni 2019 durchgeführt. Von 1931 Treffern, waren nur zehn relevant. Als mögliche Gründe der Verbesserung von Schmerz und Funktion, konnten sieben Punkte eruiert werden:

- veränderte Muskelaktivität

- gesteigerte Mobilität

- reduzierte Schmerzen

- erhöhte Druckschmerzschwelle

- verändertes spinales Gewebe

- verändertes Zentrales Nervensystem bzw. veränderte reflektorische Abläufe - korrigierte vertebrale Dysfunktion

Jedoch ist keine Erklärung einer anderen überlegen. Die Autoren empfehlen daher die Ausarbeitung eines theoretischen Rahmens, um zukünftige Studien effektiv gestalten zu können.

Quelle

Jun P, Pagé I, Vette A, Kawchuk G (2020) Potential mechanisms for lumbar spinal stiffness change following spinal manipulative therapy: a scoping review. Chiropractic \& Manual Therapies 28(15): https://doi.org/10.1186/s12998-020-00304-x.

\section{Lessons learned from cases of rib fractures after manual therapy: a case series to increase patient safety}

\section{Eine Fallserie zum Thema Patientensicherheit nach manueller bzw. chiropraktischer Therapie}

In einer Auswertung semistrukturierter Interviews, wurden von drei Chiropraktikern drei Behandlungsfälle mit Nebenwirkungen in Form von Rippenbrüchen untersucht. Die drei Patienten waren 57 Jahre und älter, davon zwei Frauen und ein Mann. Die angewandten Techniken waren verschieden: in zwei Fällen thorakal und in einem Fall lumbal. Die Rippenbrüche wurden von den Patienten als mild beschrieben. Die Chiropraktiker schließen aus der Fallserie auf folgende Empfehlungen:

1. Bestätigen und Aktualisieren von frakturbegünstigenden Faktoren

2. klare Kommunikation vor und nach der Manipulation bzw. nach einem unvorhergesehenen Ereignis

3. Erweitern der studentischen Ausbildung bezüglich des Managements unvorhergesehener Ereignisse

Quelle

To D, Tibbles A, Funabashi M. (2020) Lessons learned from cases of rib fractures after manual therapy: a case series to increase patient safety. Journal of the Canadian Chiropractic Association, 64(1):7-15. 
Herausgeber: $\quad$ Forschungsberatungsstelle (FBS) Manuelle Medizin (MM), mit Sitz am Institut für Physiotherapie am Universitätsklinikum Jena, gefördert von der Deutschen Stiftung Manuelle Medizin (www.stiftungmm.de)
Redaktion: $\quad$ Prof. Dr. U.C. Smolenski, Dr. S. Derlien,

D. Loudovici-Krug

Kontakt: $\quad$ Tel. $03641-9325238$ |

E-Mail: pt-forschung@med.uni-jena.de

\title{
Fortbildung des Landesverbandes Bayern der Deutschen Gesellschaft für Manuelle Medizin
}

\author{
MASTERKURS Manuelle Medizin 10./11.10.2020
}

\section{Thema: „HWS-Kopfgelenke mit Einbeziehung der Akupunktur"}

Liebe Kolleginnen und Kollegen,

sehr herzlich lade ich Sie zu unserer Herbstfortbildung 2020 nach Prien am Chiemsee ein.

Die HWS und die Kopfgelenke sind von zentraler Bedeutung für das Leben. Es bestehen eine Vielzahl von reflektorischen Beziehungen zum gesamten Organismus. Wir werden uns vor allem mit Manipulationstechniken, aber auch osteopathischen Techniken in diesem Bereich beschäftigen. Zusätzlich möchten wir am Sonntag auf die HWS/Kopfgelenke und einfache Akupunkturmethoden zur Therapieerleichterung für jeden (auch ohne Erfahrung) beschäftigen.

Wir freuen uns schon auf den Kurs und ein Wiedersehen.

Beste Grüße Ihr

\section{Dirk Polonius}

\section{Programm}

Samstag 10.10.2020

$09.00-09.15 \mathrm{~h}$

$09.15-10.00 \mathrm{~h}$

$10.00-10.30 \mathrm{~h}$

$10.30-11.00 \mathrm{~h}$

$11.00-13.00 \mathrm{~h}$

$13.00-15.00 \mathrm{~h}$

$15.00-16.30 \mathrm{~h}$

$16.30-16.45 \mathrm{~h}$

$16.45-18.00 \mathrm{~h}$

Sonntag 11.10.2020

$09.00-09.15 \mathrm{~h}$

$09.15-10.30 \mathrm{~h}$

$10.30-11.00 \mathrm{~h}$

$11.00-13.00 \mathrm{~h}$

$13.00-13.30 \mathrm{~h}$
Begrüßung und organisatorische Hinweise

VT Dr. Polonius: Anatomie und reflektorische Beziehungen der HWS und Kopfgelenke.

Praktisches Üben HWS Diagnostik

Pause

Praktisches Üben HWS Diagnostik und Therapie

Mittagspause

Praktisches Üben Kopfgelenke Diagnostik

Pause

Praktisches Üben Kopfgelenke Diagnostik und Therapie

Fragen und Wiederholung vom Vortag

VT Prof. Gumbiller: Einfache Akupunkturmetho-

Pause

Praktisches Üben Akupunktur

Abschlussdiskussion mit Themenfestlegung 2021 den zur Therapieerleichterung HWS/KoG

\author{
Kurslehrer: $\quad$ Prof. Gumbiller, Dr. Polonius, \\ Ort: $\quad$ Prienamed Ärztehaus \\ Harrasser Str. 6 \\ 83209 Prien \\ Teilnehmer: $\quad$ Ärztinnen und Ärzte mit abgeschlossener Weiterbil- \\ dung in „Chirotherapie“ \\ Gebühren: $\quad 300$--Euro für Mitglieder DGMM (DGMSM, MWE, \\ ÄMM)/Ö̈̈MM, \\ 350.-Euro für Nichtmitglieder
}

Reservierung in der Reihenfolge der Anmeldung. Sie erhalten eine schriftliche Bestätigung per Mail. Bitte bei der Anmeldung Angabe der Adressdaten.

Anmeldung: DGMM-Bayern@gmx.de

Save the date: Frühjahresfortbildung am 06/07.03.2021 


\section{„Den Weiterbetrieb der Klinik waren wir unseren Patienten schuldig"}

\section{Die Folgen der Corona-Krise}

Kremmen, 22. Juli 2020 Die Corona-Pandemie hat Deutschlands Krankenhäuser in den Ausnahmezustand versetzt: Kapazitäten für Covid19-Patienten wurden geschaffen, planbare Behandlungen verschoben, der Regelbetrieb vielerorts über Wochen ausgesetzt. Maßnahmen, von denen auch die Krankenhäuser der ANOA (Arbeitsgemeinschaft nicht operativer orthopädischer manualmedizinischer Akut-Kliniken) betroffen waren.

Frau Kompatzki (Pressebüro ANOA) sprach mit Dr. Jan Emmerich (Chefarzt der Klinik für Manuelle Medizin der Sana-Kliniken Sommerfeld und Leiter der Arbeitsgemeinschaft Medizin bei der ANOA) über Folgen von Corona für seine Klinik sowie für die bundesweit tätige medizinisch-wissenschaftliche Vereinigung von mittlerweile 30 Akutkrankenhäusern, die im nicht operativen orthopädisch-unfallchirurgischen, manualmedizinischen und schmerztherapeutischen Bereich tätig sind.

Herr Dr. Emmerich, was genau waren die Herausforderungen, mit denen sich Ihre Klinik sowie die anderen 29 Kliniken der ANOA durch den Ausbruch der Corona-Pandemie konfrontiert sahen?

Das Gesundheitswesen ist ja bekanntermaßen ein komplexes und hoch effizientes System: kommt es an einer Stelle zu Störungen, hat dies Auswirkungen auf viele andere Bereiche. Sämtliche Kliniken waren von heute auf morgen angehalten, neue Erlässe zum Schutz der Patienten und des Personals umzusetzen und einzuhalten. Eine logistische Herausforderung für alle Kliniken unserer Fachgesellschaft. In einigen Häusern wurde die Anzahl von Beatmungsbetten aufgestockt, vereinzelt kam es sogar zur Umwidmung von Krankenhäusern in Corona-Kliniken.

$\mathrm{Da}$ es seitens des Brandenburger Gesundheitsministeriums den Beschluss gab, die Fachkliniken primär nicht in die Versorgung von Coronapatienten einzubeziehen, konnten wir in den Sana-Kliniken Sommerfeld unsere Arbeit fortsetzen. Allerdings kam es zu erheblicher Verunsicherung bei den Patienten: So wurden gerade in der Anfangsphase der Pandemie etwa vierzig Prozent der Anmeldungen zurückgezogen. Aus

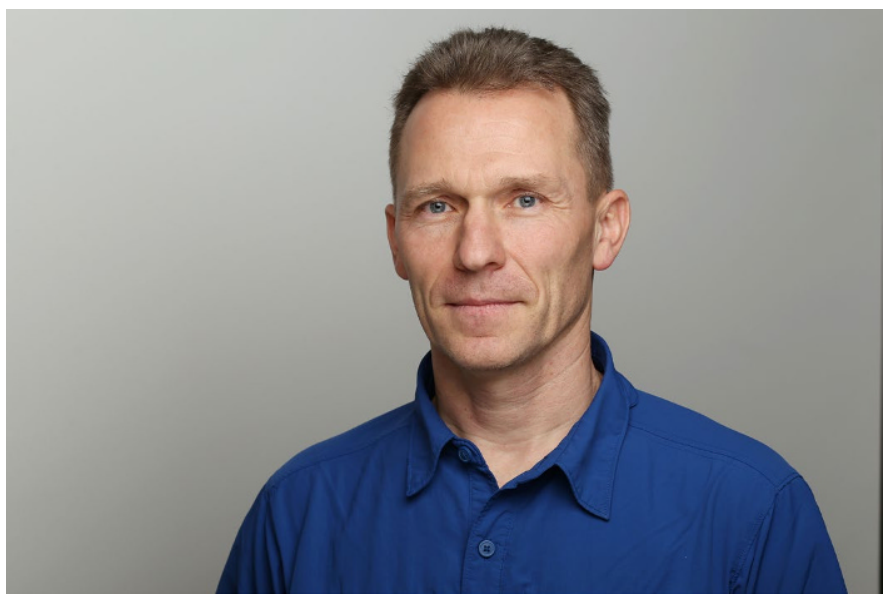

Sicherheitsgründen haben wir zudem die Aufnahmetermine einiger Patienten, die zu den Risikogruppen gehörten, von unserer Seite her aufgeschoben. Somit fiel die Anzahl unserer wöchentlichen Aufnahmen um mehr als ein Drittel. Durch diese Reduktion der Patientenzahl konnten allerdings wichtige Maßnahmen getroffen werden, um unsere Patienten vor einer möglichen Infektion zu schützen und gleichzeitig weiterhin den hohen Standard unserer multimodalen Komplexbehandlung, der im ANOA-Konzept festgehalten ist, aufrechtzuerhalten. Immerhin kam es in den vergangenen Monaten zu keinem Covid-19 Fall in Sommerfeld.

\section{Dann waren die von Ihnen durchgeführten Maßnahmen offensicht-} lich sehr wirkungsvoll. Was genau haben Sie denn bei der Patientenversorgung verändert?

Normalerweise behandeln wir in unserer Therapieabteilung alle Patienten der Kliniken am Standort, also mehr als 400 Menschen, die sich dann sowohl in den Wartebereichen begegnen als auch in einigen der Therapien aufeinandertreffen. Um das Risiko einer Ansteckung klein zu halten, war es notwendig, die Anzahl der Begegnungen auf ein Minimum zu reduzieren. Dazu haben wir unsere Patienten stationsweise in Kohorten - oder anders gesagt Familien - aufgeteilt, jeweils mit einer festen Zuordnung von Ärzten, Physiotherapeuten, Psychotherapeuten und Pflegepersonal.

\section{Dann gab es doch sicherlich Platzprobleme auf Ihren Stationen?}

Richtig. Wir brauchten zusätzliche Behandlungsräume. Um diese zu schaffen, wurden freie Patientenzimmer umfunktioniert. Das für eine befundgerechte Therapie notwendige breite therapeutische Spektrum konnten wir dadurch aufrechterhalten, dass wir zum Beispiel einige Behandlungen wie unsere Trainingstherapie nach draußen verlagert haben. Alles in allem war Kreativität gefragt: So haben wir Teile unserer Patientenedukation als Videos produziert und zusätzlich in unseren PatientenInfokanal eingespeist. Als weitere Maßnahmen haben wir 3-Bett-Zimmer zu 2-Bett-Zimmern umgewidmet, unsere Cafeteria geschlossen, die Essensversorgung in den Patientenzimmern ermöglicht und Besuche nicht mehr zugelassen. Zeitweise führten wir bei jedem Patienten, der neu aufgenommen wurde, aus Sicherheitsgründen einmalig einen Test auf COVID-19 durch.

Das klingt nach einer großen logistischen Herausforderung für Ihre Klinik. Wäre eine Schließung während der Corona-Pandemie nicht einfacher gewesen?

Einfacher vielleicht, aber keinesfalls eine Option. Chronische Schmerzerkrankungen am Bewegungssystem sind zwar nicht lebensbedrohlich, aber sie haben eine hohe Relevanz für die Betroffenen. Der Leidensdruck ist enorm und die Behandlungsnotwendigkeit bleibt natürlich bestehen, unabhängig davon, dass in der Welt Coronaviren unterwegs sind. Unsere Arbeit am Patienten ist systemrelevant, daher stand es für uns immer außer Frage, die Behandlungen fortzusetzen. Anders gesagt: Den Weiterbetrieb der Klinik waren wir unseren Patienten schuldig. Wie richtig diese 
Entscheidung war, zeigen im Übrigen auch die aktuellen Entwicklungen: Wir erleben in diesen Tagen einen wahren Ansturm von Patienten, die ihre Behandlung nachholen wollen. Die Warteliste liegt jetzt bei elf Monaten und wir bemühen uns nach Kräften, Akutpatienten zügig aufzunehmen und auch alle anderen wartenden Patienten schnellstmöglich zu behandeln. Doch unter den aktuellen Gegebenheiten müssen wir das Kohorten-Prinzip vorerst aufrechterhalten. Somit werden wir auch in absehbarer Zukunft noch nicht die Vor-Corona-Kapazitäten erreichen können.

\section{War und ist die wirtschaftliche Effizienz Ihrer Klinik unter diesen Umständen denn überhaupt gewährleistet?}

Dazu vertrete ich einen ganz klaren Standpunkt: Die Erwirtschaftung von Erträgen in der Medizin darf auch in diesen Zeiten nicht die erste Priorität haben. Es liegt auf der Hand, dass wir zurzeit nicht so effizient arbeiten können wie früher. Dazu müssten wir zum Beispiel sämtliche Zimmer vollständig belegen können. Glücklicherweise hat uns der Krankenhausrettungsschirm in dieser schwierigen Phase geholfen, den erhöhten Aufwand aufzufangen. Wir haben ihn dort genutzt, wo es zur Kompensation von Ausfällen notwendig war. Aber ich möchte nochmals wiederholen: Priorität war es auch während der Corona-Pandemie, unsere Patienten zu behandeln. Natürlich durften wir auch kein Personal verlieren. Das wäre in dieser Situation kontraproduktiv gewesen: Schließlich hat sich der Personalaufwand bedingt durch unser Kohorten-System deutlich erhöht. Da sich der Rettungsschirm verändern wird, erwarten wir in den nächsten Monaten allerdings einen steigenden finanziellen Druck durch die Zunahme der Ausfälle.

$\mathrm{Zu}$ Gute kam uns im den letzten Wochen, dass der MDK im Zuge der Pandemie die Prüfquote von 12,5\% auf fünf Prozent reduziert hat. Dadurch wurden wir von einem Teil der Bürokratie entlastet und konnten unser medizinisches Personal für erforderliche medizinische Tätigkeiten einsetzen.

Dann konnten Sie ja die Krise der vergangenen Wochen und Monate den Umständen entsprechend gut bewältigen. Wie blicken Sie und wie blickt die ANOA in die Zukunft?

Ich für meinen Teil bin optimistisch gestimmt. Die schlimmste Phase ist jetzt erst einmal vorbei und wir konnten in den Sana-Kliniken Sommerfeld einige der Sicherheitsmaßnahmen nach und nach lockern. Die Infektionszahlen in unserem Einzugsgebiet sind sehr niedrig. Natürlich bleiben wir wachsam, damit der Schutz unserer Patienten und unseres Personals jederzeit weiterhin gewährleistet ist. In den anderen ANOA Kliniken wird, wie ich weiß, ebenso verfahren. Parallel zu meinen Aufgaben in der Klinik merke ich jetzt im Übrigen auch, dass die Arbeit bei der ANOA, bei der ich im Beirat und als Leiter der AG Medizin tätig bin, wieder enorm an Fahrt gewinnt. Ganz oben auf unserer Agenda stehen die weitere Arbeit an unserem Konzept sowie die Erarbeitung und bundesweite Verbreitung unseres neuen Dokumentationsleitfadens. Außerdem sind die anstehenden Strukturprüfungen des Medizinischen Dienstes vorzubereiten, um die solide Basis für unsere weitere Arbeit zu erhalten.
Das klingt jetzt ein Stückweit nach „,back to normal“, was ja ein Zustand ist, den wir uns alle wünschen. Eine abschließende Frage: Die vergangenen Monate haben Leitung und Personal Ihrer Klinik und der anderen 29 ANOA Häuser stark gefordert. Gibt es auch etwas Positives, was Sie dieser Krise im Gesundheitssystem abgewinnen können?

In jedem Fall, schließlich hat eine Medaille immer zwei Seiten. Wir haben festgestellt, dass es allen Berufsgruppen in unseren interdisziplinären Teams - vom Arzt über den Psychologen bis hin zum Therapeuten - gelungen ist, unter den besonderen Anforderungen während der Pandemie und vor allem bedingt durch die kleineren Gruppen noch gezielter auf die Befunde der Patienten einzugehen und dabei das ANOA-Konzept optimal umzusetzen. Eine Entwicklung, die in der Konsequenz unseren bereits sehr guten Zusammenhalt in der Klinik noch weiter gestärkt hat. In der Folge waren auch die Rückmeldungen von unseren Patienten ganz besonders positiv. So wurde die verstärkte individuelle Zuwendung von nahezu allen Patienten anerkannt und außerordentlich wertgeschätzt. Diesem hohen Anspruch wollen wir, auch wenn Corona irgendwann Geschichte ist, weiterhin gerecht werden.

Sehr dankbar bin ich für die hervorragende, engagierte und konstruktive Zusammenarbeit der Mitarbeiter aller medizinischen Berufsgruppen, der Geschäftsführung und der technischen Bereiche unserer Klinik, welche diese großen Veränderungen in kürzester Zeit erst möglich gemacht haben.

Besten Dank für das Gespräch! 